\title{
LOS HORNOS DE CAL DE VALVERDE DE LEGANÉS (BADAJOZ): ESTU- DIO ARQUEOLÓGICO Y PUESTA EN VALOR ${ }^{1}$
}

Noé Conejo Delgado²

\section{Resumen:}

En este trabajo se presenta un estudio arqueológico y archivístico sobre seis hornos de cal que se conservan en Valverde de Leganés (Badajoz). Su estudio aporta datos para conocer el devenir socioeconómico de la localidad.

\section{Palabras claves:}

Hornos, Cal, Arqueología Industrial, Patrimonio Tradicional, Economía.

\section{Abstract:}

In this work, we present a archaeological and archival study about six lime's ovens what Valverde de Leganés (Badajoz) preserve in your municipal limit. This study is most important for knowing the social and economic develop of twon.

\section{Key words:}

Oven, lime, Industrial Archaelogy. Traditional Heritage, Economy.

\section{Introducción}

El principal objeto de esta comunicación es dar a conocer ciertos vestigios de arqueología industrial que posee el término municipal de Valverde de Leganés (Badajoz), en concreto, el estudio de seis hornos de cal, los cuales forman parte del vasto patrimonio vernáculo localizado en las inmediaciones de la localidad.

Debido a que estas estructuras industriales no han sido estudiadas con anterioridad, hemos llevado a cabo un trabajo de documentación completo con un rastreo bibliográfico y archivístico, con tal de hallar datos que nos pudieran aportar cronologías o particularidades de su uso, y una aproximación arqueológica mediante la toma de medidas, dibujo de alzados y plantas junto un amplio reportaje fotográfico, ya que algunas de estas estructuras se encuentran al borde de la desaparición. Por otra parte, no hemos olvidado la oportunidad de poder

1 Este pequeño trabajo de investigación ha sido financiado por Excmo. Ayuntamiento de Valverde de Leganés. Se espera poder profundizar mucho más sobre este tema con el propósito de una futura publicación más amplia. Aprovecho estas líneas para agradecer su interés, al igual que la ayuda prestada, a mi buen amigo Rafael Álvarez Fárias, quien me acompañó y colaboró en las mediciones y toma de coordenadas.

2 Universidad de Sevilla. Miembro del Grupo de Investigación "De la Turdetania a la Bética" (HUM-152) 
entrevistarnos con los descendientes de los maestros caleros, quienes durante muchos años han trabajado en torno a estas evidencias industriales y han cobrado gran importancia a la hora de rehabilitar algunos de ellos.

\section{Valverde de leganés: situación y entorno}

Valverde de Leganés es una localidad situada a unos $25 \mathrm{~km}$ al sudeste de Badajoz, formando parte de la Comarca de los Llanos de Olivenza. Presenta una población de unos 4200 habitantes y un término municipal que comprende una superficie de $72 \mathrm{Km}^{2}$. Desde un punto de vista socioeconómico, las principales fuentes de ingreso proceden del sector primario y secundario, siendo las producciones más significativas las del sector olivarero y vitícola, el porcino y sus derivados, y la fabricación del carbón vegetal. Sectores productivos que siguen siendo los mismos que en épocas pasadas, donde también cobró gran importancia el mundo trashumante, por encontrarse en sus cercanías el punto de inicio de la Cañada Real Soriano-Occidental (Conejo et Alii, 2014).

Desde un punto de vista geológico, el término municipal de Valverde de Leganés se encuentra dentro del área Olivenza - Monesterio, una franja que atraviesa toda el área suroccidental de la provincia de Badajoz. Situada en la parte central de la zona Ossa-Morena, en el entorno aparecen multitud de materiales paleozoicos, rocas ígneas y redescubrimientos terciarios y cuaternarios. A su vez, se documentan varias facies en los afloramientos, con potencias variables: como calizas y mármoles, pizarras violáceas y cuerpos graníticos postectónicos. La roca más frecuente es la caliza, de tonos grisáceos y tamaño de grano variable con varios tonos como beige, azulados, rojizos y negros (Tecminsa 2009: 269 - 271).

Tras el análisis químico efectuado sobre las rocas anteriores por parte del Proyecto de aprovechamiento industrial de las rocas calcáreas existentes en Extremadura (Recogido en Tecminsa 2009: 271 - 277), se pudo comprobar que la mayor parte de estas calizas eran favorables de aplicaciones industriales como la de producción de áridos, corrección de suelos y la fabricación de cal. Por tanto, no es de extrañar que no solo en el término municipal de Valverde de Leganés existan restos de estructuras industriales para la producción de cal, sino que tenemos constancia de que son también frecuentes en los demás términos municipales de la Comarca de los Llanos de Olivenza, al igual que en todos los términos que comprende la franja anteriormente citada.

\section{Datos históricos sobre los hornos}

En el rastreo archivístico y bibliográfico que hemos realizado para poder aportar una cronología estimada a los citados hornos, nos hemos topado con escasas 
menciones a estas estructuras industriales. No obstante, las pocas noticias documentadas son bastante interesantes para ofrecernos una visión económica de la actividad del calero en el devenir socio-económico de la localidad.

La primera noticia histórica relacionada con los hornos de cal y de la producción de esta, se encuentra contenida en la Tasación y redacción por donde se hace la obra de la Iglesia de Valverde de Badajoz y carta de fianza, documento fechado en $1547^{3}$ y parcialmente transcrito en el trabajo de Agustín Fernández Caballero (1999). Tal escritura se refiere al intento de recuperar la obra inacabada de la Iglesia parroquial de San Bartolomé, iniciada treinta años antes, y que había sido paralizada a favor de la construcción de la Iglesia-Catedral de Badajoz, Concejo de donde dependía Valverde de Badajoz, actual Valverde de Leganés. En el documento se hace referencia en varias ocasiones a cómo tanto la Parroquia y el Concejo de Valverde de Badajoz, como el maestro arquitecto, deberían preocuparse de procurar los materiales necesarios para la edificación de la Iglesia. Así en la hoja primera del documento se puede leer:

"Otrosí, han de poner el casco fenecíes, para la obra de la iglesia, y cada metro de ladrillo y cada metro de arena y cada metro de cal y cada litro de agua, por manera de todo lo demás ha de poner el que hiciere la obra, dejando estas cosas de suso que han de ser la Iglesia y el Concejo"

y en la hoja segunda del documento:

"Otrosí, ha de poner el maestro la cal y la ha de traer a la obra y mandar el maestro a cavar la arena y traerla al Concejo a la obra y de comprar el maestro el ladrillo y todo lo ha de traer el Concejo a la obra"

Ambas referencias ponen de manifiesto que existían en las zonas cercanas al municipio no solo personas dedicadas a la producción de cal, sino también los medios necesarios para fabricarla, por tanto, se puede decir que existían hornos de cal en el término de Valverde de Leganés antes del siglo XVI. Esta idea está perfectamente enlazada con la siguiente noticia que hemos podido documentar $y$ que es fechada dos siglos después.

Gracias a los Interrogatorios efectuados por la Real Audiencia de Extremadura en $1791^{4}$, sabemos de la presencia de cinco hornos de cal en el término de la localidad, pues así figura en la respuesta dada por el Concejo de Valverde de Leganés (representado por José de la Concha y Andrés Pérez Gaitán, Alcaldes ordinarios del Concejo) a la pregunta número LVI del Interrogatorio:"Si hay mi-

3 Archivo de la Catedral de Badajoz. Legajo 2. Expediente nº 50. Año 1547.

4 Las respuestas al Interrogatorio de la Real Audiencia de Extremadura se encuentran conservadas en el Archivo Histórico Provincial de Cáceres. 
nerales, de qué especie, si se benefician, o no: si hay canteras de mármol, haspe, de cal, hieso, ú otros, y si se hace uso de ellos"; siendo respondida de la siguiente manera:

"No ai en este termino minerales, canteras de mármol, jaspe, yeso ni otros, solo cinco hornos en los que se fabrica cal, que se consume en reparo de casas y otros edificios que se construien en el pueblo"

El hecho de que los hornos de cal citados en 1791 dieran cobertura a la demanda de materiales de construcción necesarios en la localidad, como por ejemplo remodelación y finalización de las obras de la Iglesia Parroquial anteriores a $1766^{5}$, prueba que el volumen de producción de éstos debió ser amplio. De todas formas, la respuesta nos puede servir para establecer que en siglos anteriores existiera un número mayor de hornos, debido a un aumento considerable de la demanda de cal por coincidir con las grandes construcciones del municipio, como la Iglesia Parroquial, las ermitas intramuros y las casas señoriales, estas últimas en gran parte desaparecidas hoy o totalmente modificadas.

Pensábamos que el Diccionario Geográfico - Estadístico de Pascual Madoz nos iba a aportar algún dato sobre este tipo de construcción, pero de no haber sido así, es probable que las familias dedicadas a la producción de cal en Valverde de Leganés por estas fechas fueran muy escasas, y que sea esta la razón por la que Madoz no lo recogiera, debido a ser unos datos que carecieran de importancia con respecto a los demás.

No obstante, sabemos a través de las entrevistas que los hornos que actualmente conocemos han estado en funcionamiento hasta hace relativamente unos 20 - 30 años, hecho que puede dificultar el aporte de una cronología por nuestra parte. De todas formas conocemos de primera mano que las remodelaciones efectuadas en la Iglesia Parroquial en los años 60 del siglo pasado necesitaron de grandes cantidades de cal (un total de $144 \mathrm{~m}^{3}$ ), que fue elaborada gratuitamente en los hornos particulares de la finca del Rebellado por cortesía de sus propietarios Sebastián García Guerrero y Amalia Marroquín Real (Fernández Caballero 1999: 215 - 221; 2010: 368).

Según los entrevistados, la falta de demanda del producto a favor de nuevas técnicas constructivas fue paulatinamente eliminando la producción, generando así la reconversión de los maestros caleros en albañiles.

5 Fecha que se toma de referencia para establecer la conclusión de la Iglesia Parroquial, como así se refleja en el Libro de visitas pastorlaes y Relatos Cronológicos del Archivo Parroquial de Valverde de Leganés. También citado en otro trabajo (Fernández Caballero 1999: 215). 


\section{Descripción de los hornos ${ }^{6}$}

Aunque en este presente solo se van a describir un total de seis hornos de cal, tenemos que hacer referencia a la existencia de otros que no son contenidos aquí por no encontrarse en el actual término municipal de Valverde de Leganés, como es el caso de los localizados en la finca el Rebellado, a unos $7 \mathrm{~km}$ al este de la localidad, que han sido referidos en el anterior punto y que se encuentran en avanzado estado de ruina. No obstante, estas construcciones presentan las mismas características generales que comparten los demás.

No podemos asegurar que los hornos de cal que vamos a describir a continuación sean los nombrados en las respuestas al Interrogatorio de la Real Audiencia. En primer lugar, no se aportan topónimos en el documento, en segundo no disponemos ni de datos suficientes ni de criterios sólidos para establecer la antigüedad de estas estructuras industriales, pues solo podemos arriesgarnos a diferenciar entre hornos antiguos y modernos en función de la acumulación de restos de hornadas que existen en sus inmediaciones. Por otra parte, topónimos como "Casa del Calero" con una localización cercana al municipio son testigos de esta misma actividad, por lo que no sería de extrañar que muchos hornos hayan desaparecido, a favor de la construcción de otros nuevos en lugares propicios para dicha actividad.

Para evitar ser redundantes, llevaremos a cabo una descripción general de los cinco ejemplos y posteriormente iremos aportando de manera individualizada sus datos específicos.

Los hornos presentan una cámara de combustión con un diámetro aproximado de entre 2,5 y 3 metros y una planta elipsoidal, aunque en algunos de ellos si hemos podido documentar plantas totalmente circulares. La cámara dispone de un alzado, a modo de chimenea, de carácter troncocónico conservado en todos los hornos, con una altura que oscila entre los 2 y 4 metros de altura. Aunque generalmente los hornos son construídos en laderas para incrustar en ella la cámara y así evitar la pérdida de temperatura en el proceso de combustión, en los hornos documentados encontramos tanto ejemplos de esta práctica como la de situarlos exentos, cubiertos solo por los desechos de anteriores hornadas, y que por sus colores blanco y gris son el elemento perfecto para la identificación de esta actividad industrial en el paisaje.

6 Aunque hay una mayor producción científica sobre la cal y sus usos, existen muy pocos trabajos sobre el proceso productivo. Se ha tomado de modelo algunos trabajos previos como Muñoz y Shnell (2006) quienes realizan un estudio similar en la provincia de Segovia y las indicaciones de Mannoni (2004: 114 y ss.) 


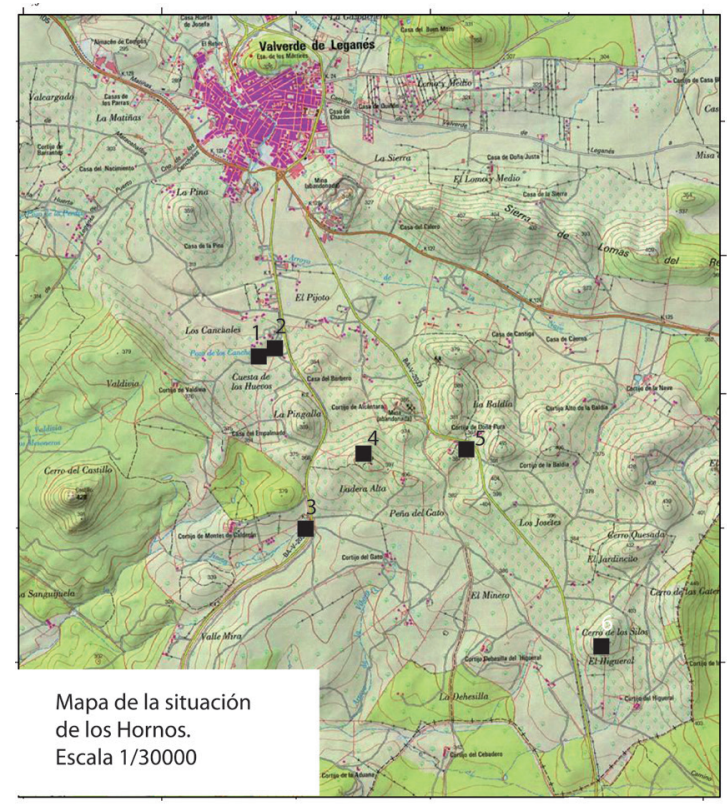

Figura 1. Mapa de la localización de los hornos de cal con respecto a Valverde de Leganés.

Debido a las altas temperaturas que se necesitan para el proceso de cocción, los materiales utilizados para la construcción, tanto de la cámara de combustión como de las paredes que ascienden, son bastante grandes ( piedras rectangulares y cuadrangulares de unos $30 \mathrm{~cm}$ de lado) identificándose granitos y cuarcitas muy resistentes al proceso de torrefacción. A su vez, estas piedras se encuentran ligadas con mortero y revestidas de una capa de barro rojizo que acaba endureciéndose de una cocción a otra. En varios hornos también hemos podido observar cómo estos paramentos son forrados con ladrillo macizo colocados de manera radial, teniendo esta práctica una función aislante y de concentración calórica seguramente.

Entre otros elementos comunes se encuentra el pequeño escalón situado en la parte baja de la cámara de combustión, cuya función era la de sostener la primera hilada de piedras que generaría la falsa cúpula sobre la que se asentarían todas las demás para su cocción. Este pequeño escalón presenta un grosor aproximado de $30 \mathrm{~cm}$ y suele colocarse a una altura de 1-1,20 metros del lecho de la cámara de combustión. Otro de los elementos es la puerta exterior que da acceso a la cámara. Generalmente suele ser un cuadrado de entre $40-60 \mathrm{~cm}$ de lado y en algunas ocasiones hemos podido observar que se ha levantado con un sistema de arquitrabe formado por granitos de gran tamaño. El túmulo formado por los deshechos de las hornadas es contenido, a veces por dos muretes a ambos lados de la puerta, acción que permite el aumento en altura del túmulo formado por las cenizas. 


\section{Cuesta de los Huevos I}

Situado $1,5 \mathrm{~km}$ al sur de Valverde de Leganés dirección Táliga, presenta una cámara de combustión de 3 metros de diámetro y un escalón para sustentar la primera hilada de $50 \mathrm{~cm}$ de grosor. La chimenea se alza dos metros sobre la cámara de combustión y está rodeada de una amplia acumulación de restos de hornadas, que son contenidos con un murete levantado con los mismos materiales que los utilizados para la chimenea (granitos y cuarcitas rectangulares regulares de 30 $\mathrm{cm}$ de ancho por 20 de alto). Dispone de una pequeña puerta de 50 por $50 \mathrm{~cm}$ orientada al norte. Recientemente ha sido consolidado para evitar su abandono. (Coordenadas geográficas: $38^{\circ} 39^{\prime} 17^{\prime \prime} \mathrm{N} / 6^{\circ} 58^{\prime} 42^{\prime \prime} \mathrm{W}$ ).

\section{Cuesta de los Huevos II}

Situado a escasos 25 metros del anterior, presenta una cámara de combustión de 2,5 metros de diámetro, escalón de $50 \mathrm{~cm}$ y chimenea de 2,5 metros de altura y 3 de diámetro. La puerta de acceso a la cámara de combustión no está cerrada como en el anterior, sino que está formada por una apertura de 2 metros de largo por $70 \mathrm{~cm}$ de ancho, también se encuentra orientada al norte. Es probable que ésta se fuera cerrando por la colocación de las piedras para su cocción. Este horno no está consolidado como el anterior, encontrándose en el interior de la cámara de combustión restos de animales muertos y basura. Los materiales constructivos son similares al anterior. (Coordenadas geográficas: $38^{\circ} 39^{\prime} 18^{\prime \prime} \mathrm{N} / 6^{\circ}$ $\left.58^{\prime} 41^{\prime \prime} \mathrm{W}\right)$.

\section{Monte Calderón}

Localizado a 2,700 km al sur de Valverde de Leganés dirección Táliga, se divisa desde la carretera que une ambas localidades. Presenta unas dimensiones muy amplias por lo que suponemos que debía de producir un gran número de $\mathrm{m}^{3}$ en cada hornada. Muestra una cámara de combustión muy pequeña, con 1,80 metros de ancho y 1,20 metros de profundidad y que parece haber sido excavada en la roca madre. El escalón para sustentar la primera hilada tiene un grosor de 30 $\mathrm{cm}$. La chimenea conserva una altura de 5 metros y un diámetro de 2,60 metros con cierta tendencia a una traza elipsoidal. El paramento de la chimenea ha sido revestido casi en totalidad con ladrillo macizo de unos $7 \mathrm{~cm}$ de grosor y a su vez, este ha sido recubierto con una fina capa de barro rojizo que se ha conservado gracias a la temperatura interior. En los muretes que dan acceso a la puerta se observa una amplia hornacina que suponemos serviría para guardar algún tipo de herramienta. La puerta se encuentra abierta como en el anterior caso y orientada al este. Por la tosquedad de los aparejos, la utilización del ladrillo y el tamaño del túmulo suponemos que es el horno más antiguo de los que hemos documentado. También ha sido limpiado recientemente pues sabíamos que durante mucho tiempo ha sido utilizado de vertedero improvisado. (Coordenadas geográficas: $\left.38^{\circ} 38^{\prime} 45^{\prime \prime} \mathrm{N} / 6^{\circ} 58^{\prime} 34^{\prime \prime} \mathrm{W}\right)$. 


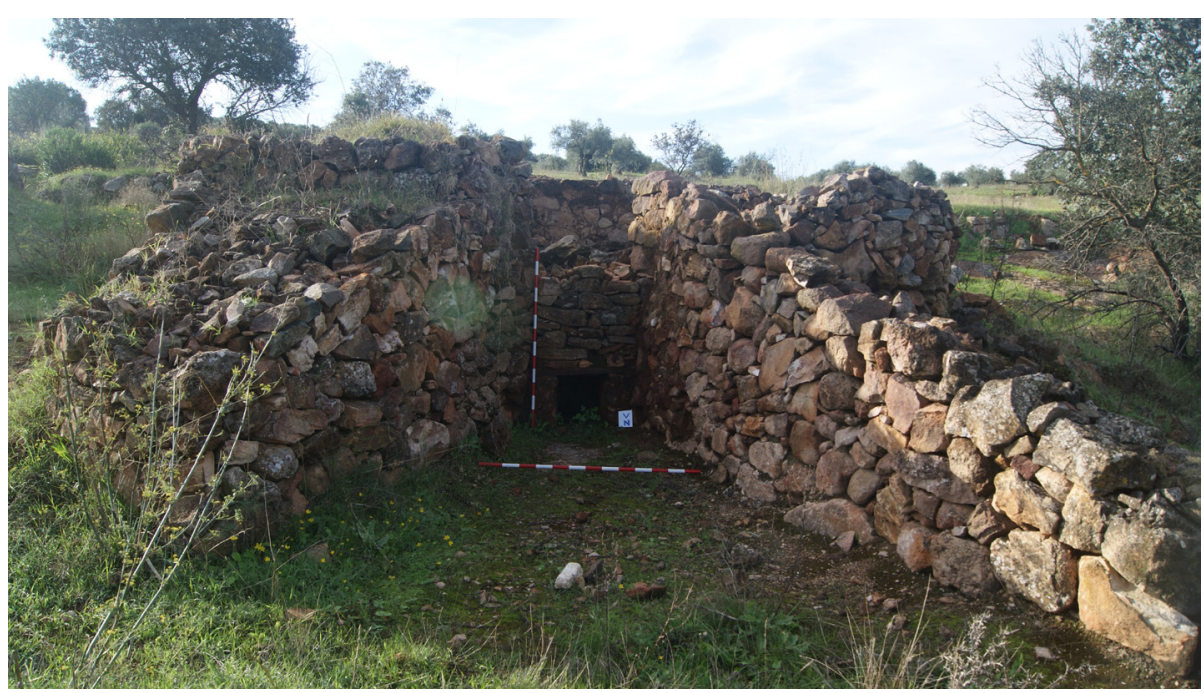

Fig. 2. Imagen del la fachada del horno Cuesta Huevos I.

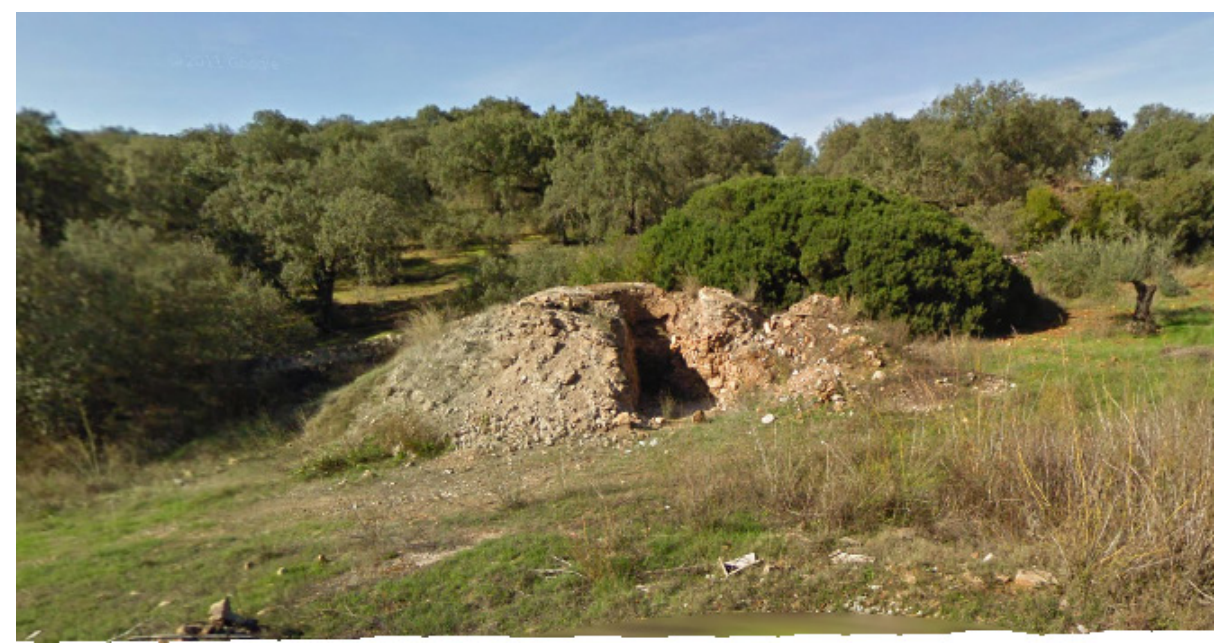

Fig. 3. Imagen del túmulo del horno Montes de Calderón.

\section{Hornos de Ladera Alta}

Formando parte de una misma estructura constructiva dos cámaras y aprovechando una ladera, se localiza en la finca Ladera Alta a unos 2,5 km al sur de Valverde de Leganés dirección Táliga, a 150 metros de la carretera. Presentan la particularidad de tener dos cámaras de combustión (A y B). En el horno denominado A, esta es mucho más amplia al disponer de una chimenea de casi 3 metros de alzado y un diámetro de 2,75 metros. No hemos podido medir el interior de la cámara porque se encuentra totalmente colmatado de restos de sucesivas 
podas vegetales. Aún así hemos podido observar la utilización de ladrillo macizo como revestimiento de la chimenea, al igual que hemos advertido en el caso de Monte Calderón. La puerta se encuentra abierta y está orientada al norte. En la parte este de la construcción se contempla una chimenea mucho más pequeña, horno B, con un diámetro de 2 metros y una altura de 2,70 metros. Tampoco hemos podido observar el interior de la cámara de combustión por encontrarse totalmente colmatada de restos orgánicos. En este pequeño horno no se han usado ladrillos como en el anterior. Su puerta está abierta, es estrecha, de unos $70 \mathrm{~cm}$ de ancho, y está orientada al este. (Coordenadas geográficas: 38 38 $54^{\prime \prime}$ $\left.\mathrm{N} / 6^{\circ} 58^{\prime} 22^{\prime \prime} \mathrm{W}\right)$.

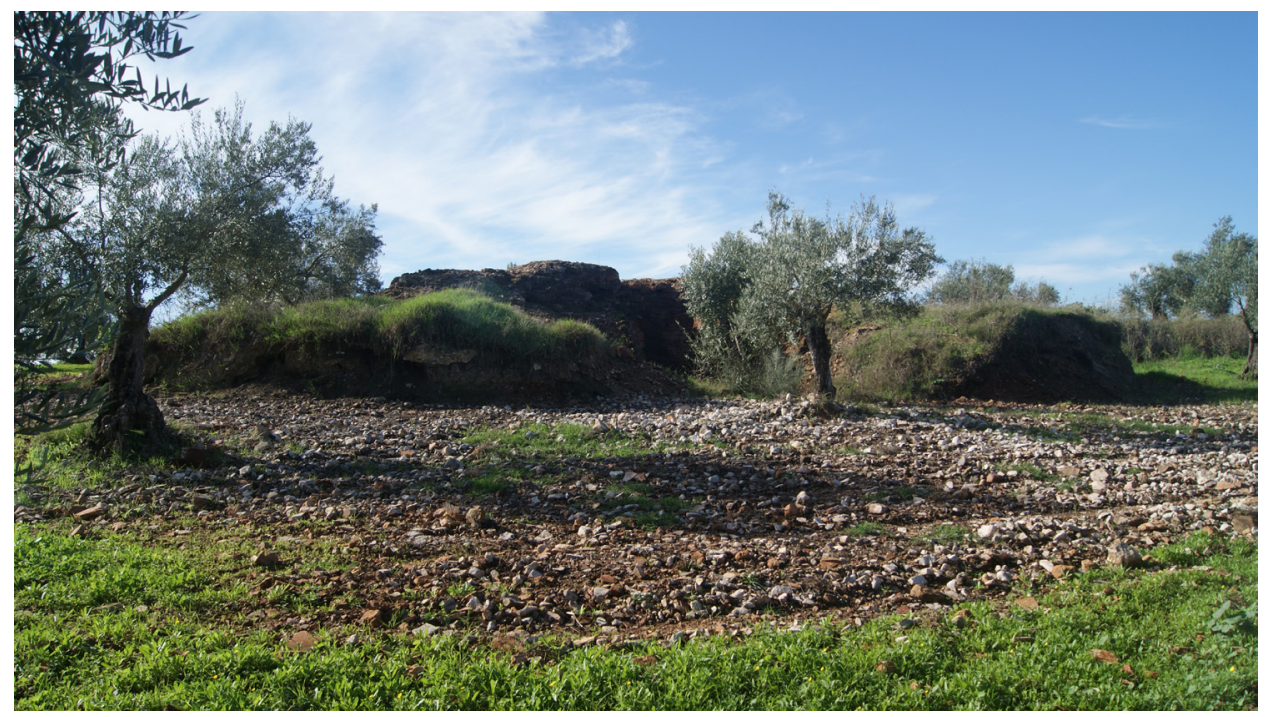

Figura 4. Imagen del túmulo que forman los dos hornos de Ladera Alta.

\section{Doña Aurora}

A 2,5 km al suroeste de Valverde de Leganés dirección Barcarrota, encontrarse enfrente de la entrada del cortijo homónimo. El lugar es un pequeño valle donde la ubicación del horno aprovecha parte de una de sus laderas. Como particularidad, a unos metros del horno se localizan los restos de una antigua vivienda que pertenecería probablemente al maestro calero. Este horno ha sido rehabilitado recientemente por lo que nos va a ser imposible dar algunas medidas exactas como por ejemplo el diámetro y altura de la cámara de combustión aunque si sabemos que su chimenea posee una altura de 7 metros y un diámetro de 3 . En lo que se refiere a la puerta, está perfectamente arquitrabada con unas dimensiones de 50 por $50 \mathrm{~cm}$ y orientada al noroeste. El túmulo es contenido por dos amplios muros que protegen la puerta y que distan uno del otro desde 1,5 metros a 5 metros. En uno de estos muros se vuelve a encontrar una hornacina similar a la documentada en Montes de Calderon, esta posee unas dimensiones reducidas: 30 por 30 
cm. Debido a ser la estructura más completa, es de esta de la que aportamos los dibujos de su alzado, sección y planta.

A pesar de haber sido rehabilitado recientemente, la estructura está siendo invadida por numerosos arbustos que con sus raíces pueden dañar la construcción. No obstante, tenemos que advertir una serie de irregularidades en la propia rehabilitación, como no haberse aplicado y utilizado los materiales adecuados para ello. El uso de cemento tipo Portland para las juntas de las piedras que conforman las paredes de la chimenea y para la fachada del horno, puede generar la caída de éstas y a su vez, la pérdida de la estabilidad de la estructura en el transcurso de los próximos años. La utilización de vigas de cemento armado para sustentar la parte superior del horno puede generar con el paso del tiempo un efecto similar al anterior. Por tanto, convendría eliminar estos elementos y utilizar el mortero de cal como tradicionalmente se ha aplicado en este tipo de estructuras.

(Coordenadas Geográficas: $38^{\circ} 38^{\prime} 56^{\prime \prime} \mathrm{N} / 6^{\circ} 57^{\prime} 46^{\prime \prime} \mathrm{W}$ ).

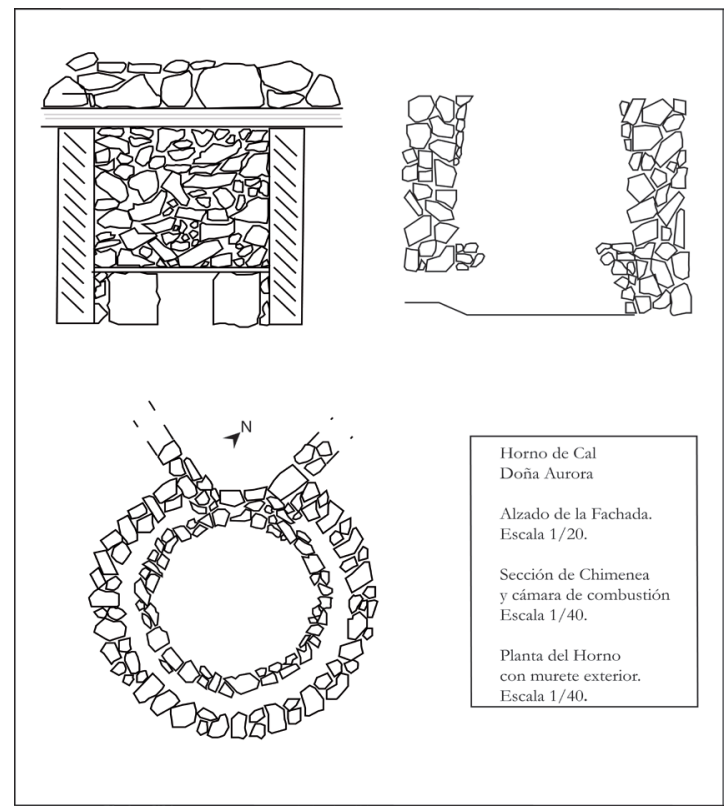

Figura 5. Alzado, planta y sección del horno de Doña Aurora.

\section{El Higueral}

Localizado en la finca homónima, a 4,7 km al sureste de Valverde de Leganés dirección Barcarrota. El horno se encuentra encajado en una ladera. Nos ha resultado complicado la medición de alguno de sus elementos, pues en la misma entrada del horno ha crecido un acebuche de numerosas dimensiones imposibilitándonos su acceso a la cámara de combustión, aunque observamos que se encuentra totalmente abierta, con una anchura de $50 \mathrm{~cm}$ y una orientación al 
oeste. No obstante examinamos una chimenea de 2,80 metros de diámetro y una altura estimada de 2,40 metros. La cámara de combustión se halla parcialmente colmatada, pudiéndose observar parte del umbral de apoyo de la primera hilada, al que calculamos un grosor de unos $30 \mathrm{~cm}$; por tanto, el interior de la cámara puede presentar un diámetro cercano a los 2 metros. (Coordenadas Geográfica: $\left.38^{\circ} 38^{\prime} 6^{\prime \prime} \mathrm{N} / 6^{\circ} 57^{\prime} 11^{\prime \prime} \mathrm{W}\right)$.

\section{Proceso de recuperación y puesta en valor}

Desde hace unos años, el Exmo. Ayuntamiento de Valverde de Leganés está realizando un inventario del patrimonio vernáculo existente en su término municipal, con la intención de llevar a cabo, en un futuro próximo, la rehabilitación y puesta en valor de estas estructuras.

Así en un primer lugar, aprovechando la existencia en el municipio de una serie de rutas senderistas que revalorizaban el medio natural y los monumentos megalíticos cercanos, se decidió configurar nuevas rutas que posibilitaran la inclusión de los hornos de cal. Esta primera toma de contacto favoreció una nueva conciencia de carácter patrimonial, al otorgar a estas olvidadas estructuras industriales un hueco en el reconocimiento del patrimonio local. Esta nueva conciencia, fruto sin duda de la socialización y patrimonialización de una tradición ancestral y cercana, permitió que varias personas, sin ánimo de lucro, prestaran sus conocimientos para rehabilitar dichos hornos, por ser ellos descendientes de los maestros caleros que habitaron en la localidad. Aún así, también hubo y hay otras personas que sin tener dichos conocimientos ofrecen su tiempo para mantener en condiciones favorables el entorno de estas construcciones.

En segundo lugar, se llevó a cabo el inventario y estudio de cada uno de estos hornos, cuyos resultados son los que se presentan en este Congreso, con el deseo de colocar próximamente cartelas explicativas donde no solo se expusieran las características de cada horno, sino también unas nociones básicas para que el visitante comprenda su funcionamiento.

Se espera que en el transcurso del 2017, todos los hornos de cal situados en el término municipal de Valverde de Leganés se encuentren totalmente rehabilitados, siendo propósito del 2017-18 la colocación de cartelas y la configuración de rutas senderistas monográficas. Este proyecto no solo se aplicará a los hornos de cal, sino que el Excmo. Ayuntamiento de Valverde de Leganés quiere hacerlo extensible, de manera paulatina, a todos los elementos de arquitectura vernácula que conserva en su término, para evitar a toda costa que se acaben perdiendo dichos ejemplos por no haberse producido previamente el proceso de socialización y patrimonialización referido con anterioridad. 


\section{Conclusiones}

El estudio de estructuras industriales de este tipo es una asignatura pendiente en el ámbito académico, siendo el uso de la cal bastante más estudiando que el proceso de su producción.

La toma de contacto con este tipo de edificación no solo nos ha servido para socializar y patrimonializar una tradición cercana en vías de extinción, sino que también nos ha permitido obtener una nueva visión en torno al devenir socioeconómico de Valverde de Leganés, pues como se ha podido comprobar en los datos históricos ofrecidos, la producción de cal en el entorno no pasó totalmente desapercibida, y si así fue, es debido al carácter tan cotidiano que tenía este producto en épocas anteriores.

En esta misma línea, no se puede dejar pasar por alto cualquier elemento de carácter tradicional que defina un oficio o una actividad productiva, pues debido a la actual revolución digital y a las constantes renovaciones de maquinarias y de instrumental que están viviendo los sectores primario y secundario, es fácil olvidarnos de una serie de herramientas que han estado vigentes durante siglos y que sin dudarlo deben ser reconocidas como patrimonio. No obstante, siempre parece más fácil la recuperación de un elemento físico, siendo mucho más complicado conservar y rescatar unos saberes centenarios que han sido transmitidos oralmente de generación en generación. Por ello, la recuperación del patrimonio intangible es un reto actualmente, pero aun se dispone de tiempo suficiente para conseguir tal objetivo, el cual no deja de ser un legado muy valioso para las nuevas generaciones. Un conocimiento vital para que estas puedan entender cuestiones tan propias del ser humano como: ¿de dónde soy? $\mathrm{O}$ ¿de dónde vengo?

\section{Bibliografía}

N. CONEJO DELGADO et Alii. (2014): "La puesta en valor de un patrimonio cultural y natural casi olvidado. Las jornadas Vive la Trashumancia de Valverde de Leganés (Badajoz)", Tejuelo: Didáctica de la Lengua y la Literatura. Educación. (Ejemplar dedicado al I Congreso Internacional sobre Educación y Socialización del Patrimonio en el Medio Rural) № Extra 9, pp. 801 815.

A. COUTELAS. Le mortier de chaux. Paris, 2009.

C. DOMERGUE: Catalogue des mines et des fondiers de la Péninsule Ibérique. Casa de Velázquez. Madrid, 1987.

A. FERNANDEZ CABALLERO: Tras las huellas de un pueblo (Valverde de Leganés: su Historia). Badajoz. 1999. 
A. FERNÁNDEZ CABALLERO: Evolución diacrónica de Valverde de Leganés y sus señoríos. Badajoz. 2010.

T. MANNONI y E. GIANNICHEDDA. (2004): Arqueología: Materias, Objetos y Producciones. Madrid.

J. M. MUÑOZ JIMÉNEZ y P. SHNELL QUIERTANT (2006): "Los hornos de cal del zancao en Vegas de Matute (Segovia)", De Re Metálica (Madrid): revista de la Sociedad Española para la defensa del Patrimonio Geológico y Minero. $\mathrm{N}^{\circ}$ 6-7. Pp. 72-82.

TECMINSA (Técnicas Mineras de Santa Marta S.L.): Recursos Minerios de Extremadura: Las rocas minerales industriales. Mérida. 2009. 
